\title{
Nivel de estudios y experiencia de las árbitras y árbitras asistentes de fútbol en España: Primera División Femenina
}

\author{
Level of studies and experience of female football referees and assistant referees \\ in Spain: Women's First Division
}

\author{
María Luisa Martín-Sánchez ${ }^{1}$, María Marín-Farrona ${ }^{1}$, Leonor Gallardo ${ }^{1}$, Jorge García-Unanue ${ }^{1}$, \\ Ángel Matute-Llorente ${ }^{3}$, José Antonio Casajús ${ }^{3}$, Javier Sánchez-Sánchez ${ }^{2}$ \\ 1 IGOID Research Group, Department of Physical Activity and Sport Sciences, University of Castilla-La Mancha, Toledo, España. \\ 2 School of Sport Sciences, European University of Madrid, España. \\ 3 GENUD (Growth, Exercise, Nutrition and Development) Research Group and IIS-Aragon, Department of Physiatry and Nursing, Faculty \\ of Health and Sport Science (FCSD), University of Zaragoza, Huesca, Spain.
}

CORRESPONDENCIA:

María Marín-Farrona

mariajesus.marin@uclm.es
CÓMO CITAR EL ARTÍCULO:

Martín-Sánchez, ML., Marin-Farrona, M., Gallardo, L., GarcíaUnanue, J., Matute-Llorente, A., Casajús, JA., \& Sánchez-Sánchez, J. (2021). Nivel de estudios y experiencia de las árbitras y árbitras asistentes de fútbol en España: Primera División Femenina. Cultura, Ciencia y Deporte, 16(50), 653-660. http://dx.doi.org/10.12800/ ccd.v16i50.1582

\section{Abstract}

The objective of this research was to analyze the sociodemographic characteristics of the referees and assistant referees belonging to the first division of women's football in Spain. The sample consisted of 45 women of Spanish nationality $(28.11 \pm 4.24$ years $)$ and was divided into referees $(n=17)$ and assistant referees $(n=28)$. An ad hoc questionnaire containing 8 items was used and analyzed social, sports and performance variables. The sample is characterized by reaching higher university training $(52.94 \%$ in female referees and $42.86 \%$ in assistant referees) and master's degree $(41.18 \%$ and $35.71 \%$ respectively) compared to professional training $(5.88 \%$ in female referees and $14.29 \%$ in assistant referees). They also showed a mean of $12.35 \pm 6.35$ years in soccer refereeing, while the assistant referees showed a mean of $8.82 \pm 4.15$ years. In relation to the training sessions, the referees showed 4-5 training sessions, while the assistant referees accumulated a total of 4 sessions per week. The female referees who participated in international matches showed a mean of $13.77 \pm 0.61$ years in arbitration, while those who did not participate in international matches had an average of $8.69 \pm 4.12$ years. The referees and assistant referees showed high levels of university academic training, evidenced a short experience in the First Division in Women's Soccer. The results show the importance of experience in refereeing performance to reach the highest level in female refereeing.

Key words: sports, competition, profile, match, socio-demographic variables.

Palabras clave: deportes, com
variables sociodemográficas. 


\section{Introducción}

El deporte ha sido un ámbito tradicionalmente masculino en todos sus niveles, al que las mujeres no han tenido un fácil acceso. A pesar de los obstáculos existentes, las mujeres han ido incorporándose a este ámbito, participando paulatinamente en un mayor número de modalidades deportivas. En concreto, el papel femenino en el ámbito del fútbol es cada vez mayor (Valenti et al., 2018). Martínez Caro (2015) reveló que entre 2000 y 2006 el número de jugadoras registradas en las 207 asociaciones de la FIFA aumentó en un 50\%. Este dato se encuentra en consonancia con un aumento sustancial en la participación y reconocimiento en los órganos internacionales (FIFA, 2016). Otras iniciativas que han favorecido este cambio fueron la resolución del Parlamento Europeo sobre las mujeres y el deporte y la creación de organizaciones específicas para impulsar la presencia de la mujer (Fernández et al., 2018).

Las mayores diferencias cuantitativas con respecto a la participación de hombres y mujeres en los diferentes estamentos del deporte se encuentran, a excepción de los deportes predominantemente femeninos, en la infrarrepresentación de las mujeres en los puestos técnicos, directivos y de gestión y en los estamentos arbitrales (Echegaray, 2016). Los primeros estudios midieron los niveles de estrés en el arbitraje en el baloncesto, y tan solo 56 de los 723 árbitros eran mujeres (Rainey \& Winterich, 1995). Estos resultados indicaron bajos niveles de estrés en los árbitros masculinos y altos niveles de estrés en el colectivo de árbitras femeninas. Otro estudio analizó la satisfacción laboral en árbitros de fútbol, a través del Cuestionario de Satisfacción de Minnesota (Weiss et al., 1967), el cual evaluaba la satisfacción general, la intrínseca (naturaleza del puesto) y la extrínseca (otros aspectos de la situación del trabajo, como las prestaciones y salario), destacando la limitada representación femenina, ya que el 98,8\% eran hombres y el 1,2\% mujeres (Çoban, 2010). En 2006 había 840.000 árbitros y árbitros asistentes registrados en todo el mundo, de los cuales aproximadamente el 10\% (85.000) eran árbitras. Otros hallazgos destacan la presencia de un total de 81 árbitras preseleccionadas para la Copa Mundial Femenina de la FIFA 2007, que representaban el 0,95\% del total de árbitras registradas en todo el mundo (Schenk et al., 2018).

El interés por analizar la evolución del perfil femenino en el arbitraje queda reflejada en el estudio llevado a cabo por Sainz de Baranda Andújar (2014), en el que se identificó un incremento en el número de mujeres en puestos deportivos a partir de un estudio sobre deportistas retiradas, directivas de clubes, árbitras y otras ocupaciones. Este aumento está acompañado de la creación de diferentes asociaciones para dar visibilidad a la mujer en el ámbito deportivo (Marugán Pintos, 2019). En este sentido, destaca la reciente creación de la Red de investigación en el arbitraje femenino $R E$ FEREENET, la cual tiene como objetivo proporcionar visibilidad, a nivel nacional e internacional, analizar la literatura existente y aportar recursos científicos, así como buscar soluciones a las principales limitaciones y problemas presentes en la actual situación del arbitraje femenino (Hernández-Martín et al., 2020).

Las investigaciones centradas en este colectivo abarcan aspectos relacionados con parámetros físicos y de rendimiento, siendo estos los más abundantes inicialmente (Bizzini et al., 2009; Castagna et al., 2018), y aquellos que analizan variables psicosociales, como nivel de empleabilidad, niveles de estudio y contexto familiar (Perreau-Niel \& Erard, 2015). Estos trataron de explorar las condiciones de acceso y empleo de los árbitros de fútbol en Francia en términos de género y nivel de arbitraje. Identificaron una diferencia entre mujeres y hombres respecto al acceso a puestos de arbitraje. Los hombres se caracterizaron por una mayor influencia del entorno social, origen deportivo familiar y experiencia en la práctica del fútbol. No obstante, respecto al colectivo femenino, las condiciones de acceso se caracterizaron por ser menos selectivas y menos discriminatorias en términos de experiencia en la práctica del fútbol. Por último, destaca como un $66 \%$ de las árbitras poseen un posgrado frente a un $59 \%$ de árbitros.

Por otro lado, Baldwin \& Vallance (2016), en su estudio sobre accesibilidad en el arbitraje, revelaron que las árbitras percibían con mayor dificultad conseguir el respeto de los integrantes de los equipos. En la misma línea Kim \& Hong (2016) exploraron los motivos por los cuales las árbitras coreanas tendían a abandonar sus puestos de arbitraje, siendo las actitudes negativas que se tienen sobre ellas el trabajo y la familia, el embarazo, el cuidado de los niños y la falta de apoyo administrativo. Reid (2016) se centró en explorar las experiencias de las árbitras que continuaban a pesar de su escasa representación, en un esfuerzo por identificar los beneficios y desafíos de ser una árbitra de fútbol en Canadá. Como resultado, la árbitra de fútbol indicó sentirse motivada principalmente para seguir arbitrando debido a su experiencia en el campo. Sin embargo, sigue mostrando sentimiento de subordinación y normalización del sexismo.

Gómez López et al., (2018) concretaron que la presencia de árbitras en el fútbol aún sigue siendo muy escasa, criticaron la inexistencia de una figura en el máximo nivel nacional, y que el mayor porcentaje de 
árbitras se encontraba en las categorías inferiores. Otros autores, como Fernández et al. (2018), describen cómo las mujeres siguen desempeñando un pequeño papel minoritario en el mundo del deporte, especialmente a medida que se asciende en el proceso de toma de decisiones a puestos de alto nivel.

La participación de mujeres árbitras en grandes eventos competitivos queda condicionada por la evolución de su papel en el arbitraje, por este motivo destacan autores que analizan su participación en diferentes eventos deportivos, como Marugán Pintos (2019), que evidencia y critica un número reducido de mujeres en los puestos técnicos de arbitraje en Tokio 2020. También destaca García-Santos et al., (2019) en un estudio realizado sobre el Campeonato Europeo Femenino Sub-16 de Baloncesto: tan solo un 33\% de la muestra eran árbitras. Por último, autores como Keller et al. (2013) estudiaron los procesos de selección femenina para diferentes eventos de gran interés, tomando como referencia lo que se venía realizando previamente en el proceso de selección de árbitros. Sin embargo, no existen evidencias científicas que analicen el perfil sociodemográfico de la árbitra de fútbol.

Echegaray (2016) invita a mejorar las políticas y programas de captación y retención o permanencia de las mujeres en puestos técnicos, arbitrales y directivos. Por todo lo descrito anteriormente, y con el fin de contribuir a una mayor participación de la mujer árbitra en los eventos deportivos, el objetivo de este estudio fue analizar las características sociodemográficas de las árbitras y árbitras asistentes pertenecientes a la primera división de fútbol femenino en España. Los resultados del presente estudio aportarán una base sobre el perfil de la mujer en el arbitraje, facilitando así el establecimiento de políticas de promoción y fidelización en el arbitraje femenino como elemento de sostenibilidad social en el fútbol.

\section{Metodología}

\section{Participantes}

La muestra de estudio estuvo compuesta por $45 \mathrm{mu}$ jeres de nacionalidad española conformada por árbitras de Primera División Femenina, donde las árbitras y árbitras asistentes revelaron una media de edad de $29.47 \pm 5.01$ y $27.28 \pm 3.44$ años, respectivamente. Las árbitras participaron voluntariamente en el estudio y las características generales de cada grupo se describen divididas por árbitras $(\mathrm{n}=17)$ y árbitras asistentes $(\mathrm{n}=28)$. La investigación cumple con el comité de ética (Comité de Investigación de Ciencias de la Salud de la
Universidad Europea, CIPI / 045/16), de acuerdo con la Declaración de Helsinki sobre los principios éticos de los médicos en la investigación en humanos. Todas las medidas fueron tomadas en la misma condición, siguiendo el mismo protocolo de actuación con cada participante.

\section{Instrumentos}

El instrumento comprendía una combinación de apartados específicos descriptivos (Anguera Argilaga \& Hernández Mendo, 2014) y contenía 8 ítems. La información se recogió mediante un cuestionario auto-aplicado (Arbinaga, Fernández-Ozcorta, HerreraMacías, \& Vela-Calderón, 2019) con variables sociales, nivel de estudios: sin estudios, E.S.O, Formación Profesional Grado Medio, Bachillerato, Formación Profesional Grado Superior, Formación Universitaria, Máster y Doctorado; y variables deportivas: árbitra o árbitra asistente; edad; años en el arbitraje; edad de inicio en el arbitraje; años de experiencia en primera división; años en llegar a primera división; número de partidos nacionales oficiales arbitrados; número total de entrenamientos semanales y duración media de cada sesión de entrenamiento. Además, se muestran las características del total de árbitras y árbitras asistentes de Primera División y su participación en Partidos Internacionales. Las variables de internacionalización han sido seleccionadas para el análisis de subgrupos, dividiendo la muestra en dos grupos en función de su participación en partidos internacionales.

\section{Procedimiento}

La herramienta se utilizó antes del inicio de la competición de manera online, a través de un enlace que se proporcionó a todas las árbitras y árbitras asistentes. Se usó un análisis de generalización utilizando un instrumento ad hoc de respuestas cerradas y abiertas (Blanco Villaseñor et al., 2014; Castellano et al., 2000). Las árbitras fueron informadas sobre el objetivo de la investigación, procedimiento y todas firmaron el consentimiento para realizar este estudio.

\section{Análisis estadístico}

Se calcularon los porcentajes, las medias y desviaciones típicas para árbitras y árbitras asistentes de Primera División de fútbol femenino español. Se comprobó la normalidad de las variables mediante la prueba Kolmogorov-Smirnov, concluyendo que todas siguen una distribución normal. Se ejecutó una prueba T para muestras independientes para analizar la existencia de 


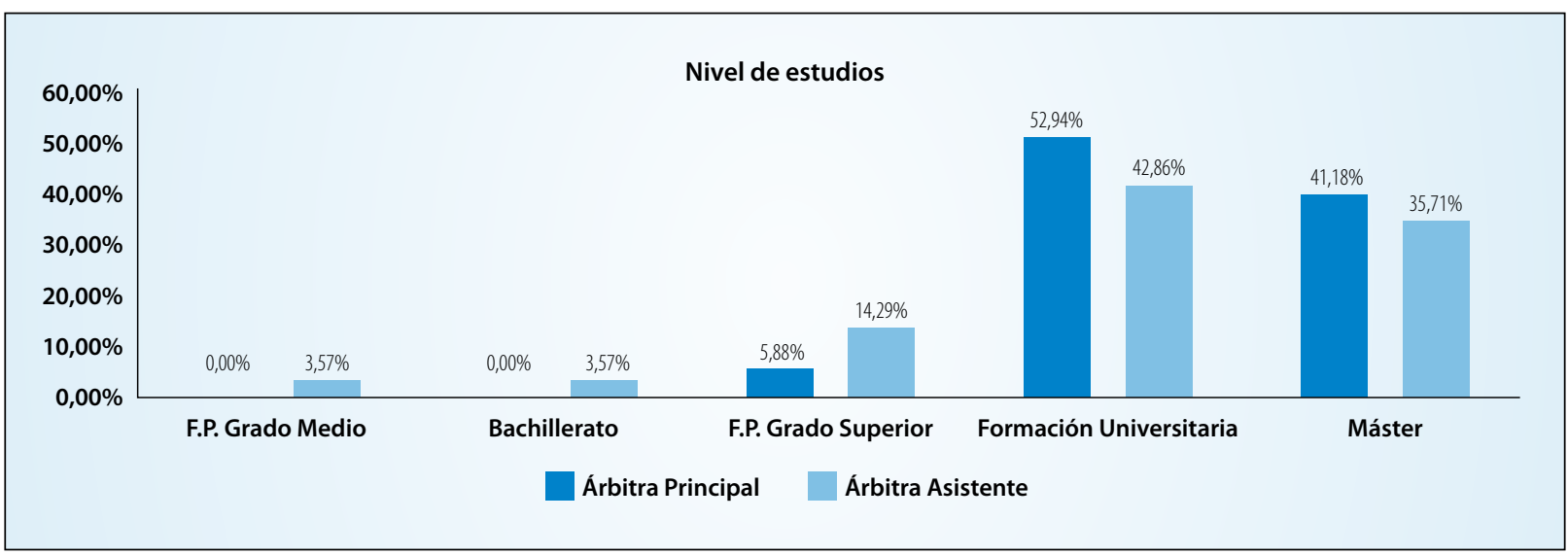

Figura 1. Porcentaje nivel estudios de árbitras y árbitras asistentes en Primera División Fútbol Femenino Español. 2019. F.P.: Formación Profesional.

Tabla 1. Características descriptivas (media y desviación típica) de las árbitras y árbitras asistentes en Primera División Fútbol Femenino Español. 2019.

\begin{tabular}{|c|c|c|c|c|}
\hline & Árbitra principal & Árbitra asistente & & \\
\hline $\mathbf{N}$ & $\begin{array}{c}17 \\
M \pm D T\end{array}$ & $\begin{array}{c}28 \\
M \pm D T\end{array}$ & Nivel significación & Tamaño efecto \\
\hline Edad (años) & $29.47 \pm 5.01$ & $27.28 \pm 3.44$ & 0.09 & 0.52 \\
\hline Años en el arbitraje (n) & $12.35 \pm 6.35$ & $8.82 \pm 4.15$ & 0.03 & 0.67 \\
\hline Edad de inicio en el arbitraje ( $n$ ) & $17.11 \pm 6.62$ & $18.46 \pm 4.62$ & 0.44 & 0.24 \\
\hline Años Experiencia en Primera División ( $n$ ) & $2.76 \pm 0.83$ & $2.36 \pm 0.87$ & 0.13 & 0.47 \\
\hline Años en llegar a Primera División (n) & $9.59 \pm 6.03$ & $6.46 \pm 3.82$ & 0.04 & 0.63 \\
\hline Número partidos nacionales oficiales arbitrados ( $n$ ) & $15.19 \pm 7.66$ & $15.63 \pm 7.69$ & 0.43 & 0.57 \\
\hline Número total entrenamientos semanales $(n)$ & $4.53 \pm 1.19$ & $4.39 \pm 0.82$ & 0.66 & 0.14 \\
\hline Duración media sesión entrenamiento (min) & $77.65 \pm 14.76$ & $68.57 \pm 14.13$ & 0.05 & 0.63 \\
\hline
\end{tabular}

M: media; DT: desviación típica.

diferencias significativas entre árbitras internacionales y nacionales. Se estableció un nivel de significación de $\mathrm{p}<0.05$. Estos datos se analizaron estadísticamente con SPSS V24.0 (SPSS Inc, Chicago, IL, USA).

\section{Resultados}

El análisis del nivel de estudios en árbitras y árbitras asistentes de Primera división (Figura 1) mostró un $52.94 \%$ de formación universitaria en árbitras y un $42.86 \%$ en árbitras asistentes. Resultados similares fueron evidenciados entre las árbitras y árbitras asistentes en formación de máster (41.18\% y $35.71 \%$ respectivamente) frente a Formación Profesional Grado Superior (5.88\% en árbitras y $14.29 \%$ en árbitras asistentes).

Las árbitras mostraron una experiencia media de $12.35 \pm 6.35$ años en el arbitraje, mientras que las árbitras asistentes reflejaron una media de $8.82 \pm 4.15$ años en el desempeño de sus funciones (Tabla 1). En cuanto a su experiencia en la máxima categoría, las árbitras asistentes mostraron una media de $2.36 \pm 0.87$ años, mientras que las árbitras revelaron una media de $2.76 \pm 0.83$ años en Primera división femenina.

Las árbitras empezaron su carrera arbitral a los 17.11 \pm 6.62 años mientras que las árbitras asistentes iniciaron su etapa en el arbitraje con una media de $18.46 \pm$ 4.62 años. Con respecto a los partidos nacionales oficiales arbitrados, las árbitras y árbitras asistentes mostraron una participación media de $15.19 \pm 7.66$ partidos y $15.63 \pm 7.69$ partidos al año, respectivamente.

En cuanto a las rutinas de entrenamiento, las árbitras evidenciaron $4.53 \pm 1.19$ sesiones de entrenamientos de media a la semana, mientras que las árbitras asistentes tuvieron $4.39 \pm 0.82$ sesiones de entrenamiento a la semana. En cuanto a la duración de estas sesiones, las árbitras y árbitras asistentes reconocieron un total de $77.65 \pm 14.76$ y $68.57 \pm 14.13$ minutos por sesión de entrenamiento, respectivamente.

En relación a las características del total de árbitras y árbitras asistentes de Primera División y su participación en Partidos Internacionales (Tabla 2), no hubo diferencias significativas en cuanto a la edad de las participantes ni los años de experiencia en primera división femenina. Sin embargo, las árbitras que par- 
Tabla 2. Características descriptivas de las árbitras y árbitras asistentes en Primera División Fútbol Femenino Español que participan en Partidos Internacionales Oficiales.

\begin{tabular}{|c|c|c|c|c|}
\hline & $\begin{array}{l}\text { Participación Partidos } \\
\text { Internacionales }\end{array}$ & $\begin{array}{l}\text { No Participación } \\
\text { Internacionales }\end{array}$ & & \\
\hline $\mathbf{N}$ & $\begin{array}{c}13 \\
M \pm D T\end{array}$ & $\begin{array}{c}32 \\
M \pm D T\end{array}$ & $\begin{array}{c}\text { Nivel } \\
\text { Significación }\end{array}$ & $\begin{array}{c}\text { Tamaño del } \\
\text { Efecto }\end{array}$ \\
\hline Edad (años) & $28.23 \pm 5.96$ & $28.06 \pm 4.11$ & 0.91 & 0.03 \\
\hline Años en el arbitraje (n) & $13.77 \pm 0.61$ & $8.69 \pm 4.12$ & $0.00 *$ & 2.15 \\
\hline Edad de inicio en el arbitraje (n) & $14.46 \pm 7.21$ & $19.37 \pm 3.80$ & $0.01 *$ & 0.89 \\
\hline Años Experiencia en Primera División (n) & $3.08 \pm 0.61$ & $2.28 \pm 0.84$ & $0.00 *$ & 1.10 \\
\hline Años en llegar a Primera División (n) & $10.69 \pm 5.85$ & $6.41 \pm 4.01$ & $0.01 *$ & 0.87 \\
\hline Número total entrenamientos semanales & $4.69 \pm 1.13$ & $4.34 \pm 0.89$ & 0.14 & 0.35 \\
\hline Duración media sesión entrenamiento (minutos) & $68.08 \pm 12.64$ & $73.59 \pm 15.62$ & 0.27 & 0.39 \\
\hline
\end{tabular}

* Diferencias significativas para p<0.05. M: media; DT: desviación típica.

ticiparon en partidos internacionales mostraron un mayor número de años de experiencia en el arbitraje (+3.53; IC95\%: 0.035 a 7.10; p <0.05) en comparación a las árbitras que no participaron en competiciones internacionales.

Las árbitras internacionales evidenciaron un inicio significativamente más temprano en el arbitraje (14.46 \pm 7.21 años) en comparación a las árbitras que todavía no habían alcanzado ese rango (19.37 \pm 3.80 años; $\mathrm{p}<0.05)$. Las árbitras con participación en partidos internacionales tuvieron una experiencia mayor en Primera División (3.08 \pm 0.61 años) con respecto a árbitras que no participaron en partidos internacionales $(2.28 \pm 0.84$ años; $\mathrm{p}<0.05)$. En cuanto a las rutinas de entrenamiento, no hubo diferencias significativas entre las árbitras internacionales y el resto ( $p>0.05)$.

\section{Discusión}

La presente investigación trata de aportar información sobre el perfil sociodemográfico de mujeres árbitras pertenecientes a la Primera División de fútbol Femenina que participan en los eventos competitivos de España. Son pocos los estudios que han centrado su interés en las mujeres árbitras, en concreto de esta modalidad deportiva, a pesar de tener un papel tan importante en este deporte y en los eventos deportivos (Kordi et al., 2013). Las áreas más investigadas dentro del arbitraje son aquellas que analizan parámetros de rendimiento físico tanto en hombres y mujeres, aunque las mujeres siguen sin ocupar un papel relevante en los diferentes análisis (Bizzini et al., 2009).

Los principales resultados muestran como las árbitras asistentes (42\%) y árbitras (52\%) tienen una formación predominantemente universitaria. De las que han realizado estudios universitarios, un $36 \%$ de árbitras asistentes, y un $41 \%$ de árbitras han realizado máster universitario. Estos resultados reflejan una diferencia considerable con los valores obtenidos por $\mathrm{Vi}$ llalobos et al. (2002), donde tan solo un 9\% de la muestra compuesta por 58 árbitros de fútbol de primera y segunda división poseía estudios universitarios, por lo que, en comparación con los datos obtenidos en la presente investigación, se observa un aumento significativo en la formación de estas profesionales. En este mismo estudio, se analizó el número de árbitras que compaginaban estudios con sus labores de arbitraje, siendo tan solo un $5 \%$ de la muestra el que compaginaba ambas labores. También está aumentando la literatura que analiza la accesibilidad, empleabilidad y diferentes variables psicológicas en el arbitraje. Por ejemplo, Çoban (2010) utilizó el cuestionario de Satisfacción de Minnesota (MSQ; Dawis, Weiss, Inglaterra, y Lofquist, 1967) para identificar los niveles de satisfacción en el trabajo. En este estudio no se encontraron diferencias estadísticamente significativas entre los árbitros en términos de nivel de educación, y antecedentes deportivos.

Por otro lado, se analizaron variables relacionadas con la edad de las árbitras. Nuestra muestra tiene una media de edad de 29,47 \pm 5,01años para árbitras y 27,28 $\pm 3,44$ años para árbitras asistentes, por lo que se trata de una muestra relativamente joven. Este dato podría explicarse por los hallazgos encontrados por Echegaray (2016), quien explica que el principal obstáculo personal para las mujeres en el desarrollo de su carrera como árbitras es la familia, lo que hace que abandonen la práctica del arbitraje en una edad temprana. Al tratarse de una actividad que exige una gran inversión de tiempo, se suelen desencadenar conflictos personales, ya que sienten que no pueden compatibilizar las exigencias de este rol con sus responsabilidades en la familia, por lo que es importante atender a la edad, ya que entender sus necesidades psicológicas ayuda a diseñar una formación y planes de promoción 
más acorde a sus demandas específicas. En relación a la edad de inicio en el arbitraje, se observa un inicio más tardío en comparación a las modalidades deportivas como el fútbol, que suelen iniciarse a los 6-8 años aproximadamente (Lapresa et al., 2016). Dado los requerimientos tanto físicos, técnicos y tácticos del arbitraje, se observan edades de inicio de las árbitras demasiado tardías. Estos autores exponen que comenzar la especialización a edades más tempranas mejora el rendimiento en el futuro, ya que una edad de inicio tardía limita las posibilidades de proyección deportiva. En otro estudio se establece un criterio temporal de 11 años a partir de las edades de inicio para determinar la llegada al éxito en el arbitraje (Gomà-i-Freixanet et al., 2020). Este valor no discrepa en exceso de los resultados obtenidos en nuestra muestra, ya que las árbitras tardan una media de 9,59 \pm 6,03 años en llegar a primera división. En el caso de las árbitras asistentes, esta media es inferior, de 6,46 \pm 3,82 años.

Çoban (2010), tras su investigación sobre variables psicosociales en el arbitraje, incide en apoyar a los árbitros más jóvenes y menos experimentados, dado el potencial observado en éstos. Estos resultados se pueden comparar con los valores obtenidos por Villalobos et al., (2002), en donde un 59\% de los árbitros analizados tenían más de 7 años de experiencia, mientras que nuestra muestra representa más años de media en el arbitraje, siendo unos $12,35 \pm 6,35$ años para árbitras, y 8,82 $\pm 4,15$ años para árbitras asistentes. En este sentido, observamos cómo la experiencia adquiere un papel muy importante, dado que se trata de personas de edad joven-adulta y, por lo tanto, sus características personales, sociales y emocionales están más definidas.

Del total de la muestra, 13 participantes intervienen en partidos internacionales, con una media de 13,77 $\pm 0,61$ años en el arbitraje, mientras que un total de 32 árbitras no participan en competiciones internacionales. Estas últimas tienen una experiencia media de 8,69 \pm 4,12 años en el arbitraje. Estas diferencias resaltan la importancia de un inicio temprano para acumular horas de práctica deliberada que faciliten la adquisición de las capacidades necesarias para un adecuado desempeño arbitral. Desde una perspectiva histórica, el primer Mundial Femenino de la FIFA tuvo lugar en China en 1991, mientras que el primer Mundial Masculino de la FIFA se celebró por primera vez en 1930, en Uruguay. Por lo tanto, dado que el fútbol internacional femenino tiene una historia más corta y éste sigue evolucionando en todos los aspectos del juego (Bizzini, Junge, Bahr, \& Dvorak, 2009), se espera observar un aumento en la participación de las mujeres en los eventos que incluyan partidos internacionales, ya que son numerosos los autores que pro- mueven la participación de las mismas en el arbitraje (Hernández-Martín et al., 2020). Autores como Bizzini \& Dvorak (2015) inciden en que las mujeres tienen que estar preparadas específicamente para actuar en un nivel élite.

Podemos concluir que la información existente sobre el perfil sociodemográfico de la mujer en el arbitraje es muy reducida, la mayoría de las investigaciones analizan parámetros de rendimiento y, por lo general, la muestra suele estar compuesta por un mayor número de hombres que de mujeres. Gómez López et al., (2018) realizaron una revisión sistemática de la literatura existente sobre el estrés y/o la ansiedad en el arbitraje en deportes de equipo. De nuevo este análisis recogía información predominantemente del arbitraje masculino. Por este motivo sería interesante incluir futuras líneas de investigación el análisis de variables psicológicas que definan el perfil de las árbitras, así como analizar la satisfacción laboral en la muestra analizada y compararla con los niveles educativos de la misma. Del mismo modo, conocer cuáles son los conocimientos que las árbitras consideran necesarios para mejorar el desempeño arbitral y compararlos con los resultados obtenidos por Villalobos et al., (2002) podría ser útil para identificar diferencias entre los dos géneros y así poder diseñar una formación específica e integral acorde a las necesidades de las árbitras femeninas. La importancia de indagar en esas características radica en que se podrían conocer los aspectos deseables para el adecuado desarrollo y sostenibilidad de la labor arbitral de la mujer en eventos deportivos. Los resultados obtenidos revelan una experiencia en primera división femenina de 2,76 $\pm 0,83$ años para árbitras y 2,36 \pm 0,87 para árbitras asistentes, por lo que el fomento de la continuidad, promoción y desarrollo del arbitraje femenino permitirá incrementar este resultado.

Este estudio presenta limitaciones, como son la escasez de variables sociodemográficas, debido a que se trata de un estudio piloto. Sin embargo, es el primer estudio que incide sobre el perfil sociodemográfico dentro del arbitraje femenino y sienta las bases para continuar investigando en esta línea de estudio.

Esta investigación puede dar una visión muy práctica para variables sociodemográficas en árbitras y árbitras asistentes de fútbol femenino, conociendo qué puntos fuertes deben reforzarse y qué puntos débiles deben mejorarse para que la calidad del arbitraje crezca y, con ello, buscar una profesionalización de éstas. Para futuros estudios, la incorporación de más variables sociodemográficas, como el nivel socioeconómico, estado civil o el número de hijos que tienen las árbitras y árbitras asistentes, sería recomendable. Además, el 
conocimiento sobre las posibles barreras que la mujer puede encontrar en sus inicios en el mundo del arbitraje del fútbol femenino en España permitirá un mayor desarrollo del arbitraje femenino en este deporte.

\section{Conclusión}

Esta investigación se centra en analizar las características sociodemográficas de árbitras y árbitras asistentes pertenecientes a la primera división de fútbol femenino en España. Los resultados evidencian altos niveles de formación académica universitaria tanto en árbitras como árbitras asistentes. Se evidencia una media de edad joven en árbitras y árbitras asistentes en comparación a los árbitros, así como una corta experiencia en Primera División en Fútbol Femenina. Por último, los resultados resaltan la importancia de los años de experiencia en el desempeño arbitral para alcanzar el máximo nivel en el arbitraje femenino.

\section{Agradecimientos}

Los autores quieren agradecer a las árbitras de fútbol femenino español su colaboración y contribución en esta investigación y al Comité Técnico de Árbitros de la Real Federación Española de Fútbol por su apoyo. 


\section{BIBLIOGRAFÍA}

Anguera Argilaga., María Teresa., \& Hernández Mendo, Antonio. (2014). Metodología observacional y psicología del deporte: estado de la cuestión. Revista de Psicología del Deporte, 2014, 23(1), 103-109.

Arbinaga, Félix., Fernández-Ozcorta, Eduardo José., Herrera-Macías, Pedro Pablo., \& Vela-Calderón, Diego. (2019). Burnout syndrome and resilience in soccer and basketball referees. Revista de Psicologia del Deporte, 28(2), 23-32.

Baldwin, C., \& Vallance, R. (2016) Rugby Union referees' experiences with recruitment and retention. Journal of Qualitative Research in Sports Studies, 10(1), 255-268.

Bizzini, Mario., Junge, Astrid., Bahr, Roald., \& Dvorak, Jiri. (2009). Female soccer referees selected for the FIFA Women's World Cup 2007 survey of injuries and musculoskeletal problems. British journal of sports medicine, 43(12), 936-942. DOI: 10.1136/bjsm.2008.051318

Bizzini, Mario, Junge, Astrid, Bahr, Roald, \& Dvorak, Jiri. (2009). Injuries and musculoskeletal complaints in referees-a complete survey in the top divisions of the swiss football league. Clinical journal of sport medicine, 19(2), 95-100. DOI: 10.1097/JSM.0b013e3181948ad4

Bizzini, M., \& Dvorak, J. (2015). FIFA 11+: An effective programme to prevent football injuries in various player groups worldwide - A narrative review. British Journal of Sports Medicine, 49(9), 577-579. DOI: 10.1136/bjsports-2015-094765

Blanco Villaseñor, Ángel., Castellano, Julen., Hernández Mendo, Antonio., Sánchez-López, Carmen Rosa., \& Usabiaga, Oidui. (2014). Aplicación de la TG en el deporte para el estudio de la fiabilidad, validez y estimación de la muestra. Revista de psicología del deporte, 23(1), 0131-0137.

Castagna, C., Bizzini, M., D’Ottavio, S., \& Póvoas, S. (2018). Sex differences in aerobic fitness in top-class soccer referees. The Journal of Strength \& Conditioning Research, 32(11), 3216-3221. DOI: 10.1519/ JSC.0000000000002292

Castellano, Julen., Mendo, Antonio Hernández., De Segura, Pedro Gómez., Fontetxa, Edorta., \& Bueno, Igor. (2000). Sistema de codificación y análisis de la calidad del dato en el fútbol de rendimiento. Psicothema, 12(4), 635-641.

Coban, B. (2010). An evaluation of the job satisfaction levels of Turkish provincial football referees. Social Behavior and Personality: an international journal, 38(9), 1153-1165. DOI: 10.2224/sbp.2010.38.9.1153

Echegaray, A. A. (2016). Obstáculos psicosociales para la participación de las mujeres en el deporte como entrenadoras y árbitras. Universidad del País Vasco-Euskal Herriko Unibertsitatea.

Fernández, Dolores., Olid, Clara., \& Torregrossa, M. (2018). Mujeres y árbitras: historias de vida de una doble minoría en el deporte. Revista de Psicología del Deporte, 27(2), 272.

García-Santos, D., Pino-Ortega, J., García-Rubio, J., Vaquera, A., \& Ibá ñez, S. J. (2019). Internal and external demands in basketball referees during the U-16 european women's championship. International journal of environmental research and public health, 16(18), 3421. DOI 10.3390/ijerph16183421

Giske, Rune., Haugen, Tommy., \& Johansen, Bjørn Tore. (2016). Training, mental preparation and unmediated practice among soccer referees: An analysis of elite and sub-elite referees' reported practice. International Journal of Applied Sports Sciences, 28(1), 31-41.

Gomà-i-Freixanet, M., Pla-Cortés, J., \& Avilés-Antón, Ó. (2020). Perfil diferencial de personalidad de los árbitros de élite del baloncesto es pañol. Cuadernos de Psicología del Deporte, 20(1), 1-9. DOI: 10.6018/ cpd.375641
Gómez López, M., Alfaro, E., \& Vázquez, B. (2018). Seminario “mujer y deporte" INEF-Madrid. El acceso de las mujeres al deporte profesional: el caso del fútbol. Jornadas nacionales (Madrid, 19 y 20 de abril de 2018). FEMERIS: Revista Multidisciplinar de Estudios de Género, 3(2), 178. DOI: 10.20318/femeris.2018.4325

Hernández-Martín, A., Colino, E., León-Jiménez, M., Felipe, J-L., Sánchez Sánchez, J., Manzano-Carrasco, S., Marín-Farrona, M., MartínSánchez, M-L., Gallardo, L., \& García-Unanue, J. (2020). Arranca con rotundo éxito la red de investigación 'Mujer y arbitraje en fútbol. Refereenet'. Deporcam, 46.

Keller, D. I., Bizzini, M., Feddermann, N., Junge, A., \& Dvorak, J. (2013). FIFA women's world cup 2011: pre-competition medical assessment of female referees and assistant referees. British Journal of Sports Medicine, 47(3), 179-181. DOI: 10.1136/bjsports-2012-091436

Kim, M. C., \& Hong, E. (2016). A red card for women: female officials ostracized in south korean football. Asian Journal of Women's Studies, 22(2), 114-130. DOI: 10.1080/12259276.2016.1168156

Kordi, R., Chitsaz, A., Rostami, M., Mostafavi, R., \& Ghadimi, M. (2013). Incidence, nature, and pattern of injuries to referees in a premier football (soccer) league: a prospective study. Sports Health, 5(5), 438441. DOI: $10.1177 / 1941738113481428$

Lapresa, D., Arana Idiakez, J., Anguera Argilaga, M. T., Pérez Castellanos, J. I., \& Amatria, M. (2016). Application of logistic regression models in observational methodology: game formats in grassroots football in initiation into football. Anales de Psicología, 32(1), 288-294. DOI: 10.6018/analesps.31.3.186951

Martínez Caro, E. (2015). Gestión de la calidad de los servicios de los clubes de fútbol base. Universidad de Murcia.

Marugan Pintos, B., S Marín García, J., Martínez Ferreiro. (2019). El de porte femenino, ese gran desconocido. Universidad Carlos III de Madrid.

Perreau-Niel, A., \& Erard, C. (2015). French football referees: an exploratory study of the conditions of access and employment for referees in terms of level and gender. Soccer \& Society, 16(1), 1-16. DOI: 10.1080/14660970.2012.627168

Rainey, D., \& Winterich, D. (1995). Magnitude of stress reported by basketball referees. Perceptual and Motor Skills, 81(3), 1241-1242. DOI 10.2466/pms.1995.81.3f.1241

Reid, K. (2016). The untold stories of women soccer referees (Doctoral dissertation, Université d'Ottawa/University of Ottawa). DOI: $10.20381 /$ ruor-280

Schenk, K., Bizzini, M., \& Gatterer, H. (2018). Exercise physiology and nutritional perspectives of elite soccer refereeing. Scandinavian journal of medicine \& science in sports, 28(3), 782-793. DOI: 10.1111/ sms.12989

Sainz de Baranda Andújar, C. (2014). Las mujeres en la prensa deportiva: dos perfiles. Cuadernos de psicología del deporte, 14(1), 91-102.

Valenti, M., Scelles, N., \& Morrow, S. (2018). Women's football studies: an integrative review. Sport, Business and Management: An International Journal, 8(5), 511-528. DOI: 10.1108/SBM-09-2017-0048

Villalobos, D., Ugarte, O., \& Guillen, F. (2002). Situación actual y expectativas del árbitro de fútbol profesional. Kinesis, 33, 17-24.

Weiss, D. J., Dawis, R. V., England, G. W., \& Lofquist, L. H. (1967). Manual for the Minnesota Satisfaction Questionnaire: Minnesota studies in vocational rehabilitation. Minneapolis: Industrial Relations Center, University of Minnesota. 